\title{
The effect of improving some visual abilities on the level performance of Fireman carry for wrestlers
}

\section{Dr / Amgad Zakaria Ahmed Abdel El-all}

Dr / Emad Sabry Saleep Saad

\section{Introduction and research problem:}

The visual system efficiency for athletes in most sports activities essential and active role in the success of many of the sports performances during the processes of training and competition, which led to the attention of researchers for the study of the most important ways and means to improve the visual abilities of athletes, according to the requirements of each sports activity.

Isabel refer to (2001) that sports scientists and coaches are looking for modern methods and programs that aim to improve athletic performance and gain a competitive advantage, and visual training one of known technology in the field of sports, where it is a recurring series of eye exercises in order to improve the visual basic functions, which are important for athletes in all competitive sports. (18: 203)

El said Ali, faika-Bader (2001) that the visual system in humans consists of eyes and visual nerves who go out of the eyes retina where they meet at the intersection point which is not the connection point, but only a transit point where all the optic nerve take another name called a visual stream which moving after the point of intersection to the other side in the cerebral cortex, which is the opposite point of the eye from which the optic nerve. $(4: 20)$

\section{As}

seen

Zaki

Mohammed (2002) the sense of sight is the sensory element for vision and select visual distances, and the presence of both eyes over the horizon of vision and field of vision, and the importance of the visual sense in the sport can be divided into two categories the first for training and the second for player, during the game the visual play an important role in the performance skills by that way the player can knows his place for the competitor and can determine the movements that can be kind of performance, and the 
movements of the opponent being able to take the appropriate position for that attack or defense. (12: 270)

Layla refaat (2009) that the visual training in the field of sports is a relatively small area in athletic performance system but great importance, where the interest in them has become a large and increasingly active in recent periods. (7:318)

Susanna (2003) visions exercises did not have a place in the daily preparation of athletes, and coaches are training by visual vision unintentionally, but research has demonstrated the importance of the visual capabilities of the performance of the athlete, also it revealed that the athletes have visual capabilities are high compared to non-athletes, it has made many researchers to verify the possibility of training these visual capabilities, and some studies have indicated the presence of a positive outcome of the training. (20:54)

The researchers also point out that wrestling is one of the sports harvester medals in regional and international tournaments and competitions that have special requirements due to the nature of the competition between the players equal in age and weight process where each is trying to thwart attempts by rival attack to get as many points by using the limbs and trunk and directing this attack according to international law governing the wrestling matches.

In addition to the specific surface area by 9 meters actual competition. in the case of exit wrestler is exposed to alarm the corresponding points are added to the balance of the opponent, and the wrestler deals during the match with a moving target is the opponent and moving in different places of the mat, shall be the competitor in the middle of the mat and also be on the edge of the mat, and the presence of competitor in any of these places requires an act tactically differs it from other places, which calls for the importance of directing the player and the senses, especially the sense of sight to competitor and follow his movements as well as the whereabouts of the player on the mat so that it can be fully prepared to carry out attacks accurately and effectively, which calls for the importance of identifying the player before the implementation of the 
various attacks of the appropriate place to carry out these attacks.

The researchers also refers to the skill of fireman carry is one of the attack skills upon which the wrestler to get as many points during the matches, and this has emerged over the last ten years during international and world championships, where freestyle wrestlers winners in advanced centers in these championships are characterized by mastering the skill of fireman carry.

The researchers attributed to a rise in the intensity of competition and the high level of performance of the speed of implementation of attack and defensive and counter attack skills and ability to integrate between defense and attack and optimization for each potential and capabilities wrestlers (technique and tactical).

Also the researchers during the follow-up to the wrestling championships in Sharkia governorate deficiencies in the performance of the skill of fireman carry with (Grand technique) during the matches represented in the lack of success of many skills in achieving conditions of grand technique, in addition to the withdrawal of several wrestlers out of the mat borders and a lot of them on the warnings and sanctions during the games and in spite of these players marked a high level of physical and skill level of efficiency.

The researchers attributed this problem to the existence of deficiencies in the level of some of the visual capabilities of the wrestler may be a major and influential reasons for the effective performance during the wrestling matches, and through the reference survey studies linked it is clear that there is no study in the limits of science researchers addressed this problem, study and research prompting researchers to address this problem, such as research and study with the aim of some visual capacity development and to identify the impact on the level performance of the skill of a fireman carry in freestyle wrestling.

Aim of the research: The research aims to identify the impact of the development of some of the visual abilities to level performance of fireman carry skill for wrestlers.

\section{Research hypotheses:}

1- There are statistically significant differences between 
the averages of pre and after measurements in some visual Abilities and level performance of fireman carry skill for the experimental group in favor of the after measurement.

2 - There are statistically significant differences between the averages of after measurements for the experimental and control groups in some visual Abilities and level performance of fireman carry skill and in favor of the experimental group.

3. There are differences in Improvement ratios between experimental and control groups in some visual Abilities and level performance of fireman carry skill and in favor of the experimental group.

\section{The plan and the procedures} of the Research:

Research procedures: The researchers used the experimental methodology using a pre- measurements and after measurements of two group(experimental\&control group).

The research sample: The sample was chosen with random way from wrestling team of Shiba youth Center el sharkia Region Wrestling, and the number of the sample (29) wrestlers, was selected (5) wrestlers for survey study to become a number of sample (24) wrestlers were divided randomly into two groups experimental and control, and the number of each of them (12) wrestlers in both groups.

\section{Table (1)}

Statistical characterization of the sample research in growth variables $\mathbf{n}=\mathbf{2 9}$

\begin{tabular}{l|l|c|c|c|c|c}
\hline \hline Sr. & $\begin{array}{l}\text { The } \\
\text { variables }\end{array}$ & $\begin{array}{l}\text { Unit of } \\
\text { measuring }\end{array}$ & mean & Deviation & Median & skewness \\
\hline \hline 1 & age & Year & 19.41 & 3.27 & 20.20 & -0.72 \\
\hline 2 & weight & $\mathrm{Kg}$ & 75.90 & 3.97 & 75.00 & 0.68 \\
\hline 3 & Tallness & $\mathrm{Cm}$ & 175.86 & 3.80 & 176.00 & -0.11 \\
\hline \hline
\end{tabular}

Table (1) that the skewness values are limited between ($0.72: 0.68$ ) and all values between \pm 3 , which refers to the homogeneity of the research sample. 
Table (2)

Statistical characterization of the sample research in some visual abilities $\mathbf{n}=\mathbf{2 9}$

\begin{tabular}{|c|c|c|c|c|c|c|}
\hline Sr. & $\begin{array}{l}\text { The } \\
\text { variables }\end{array}$ & $\begin{array}{l}\text { Unit of } \\
\text { measuring }\end{array}$ & mean & Deviation & Median & skewness \\
\hline 1 & $\begin{array}{l}\text { Visual } \\
\text { reaction time }\end{array}$ & marks & $10 . \mathrm{H}$ &.$\leqslant 9$ & $10 \ldots$ & r.r. \\
\hline 2 & $\begin{array}{l}\text { Peripheral } \\
\text { vision (right } \\
\text { eye) }\end{array}$ & marks & Y.YI &.$\leqslant 1$ & r... & 1.01 \\
\hline 3 & $\begin{array}{l}\text { Peripheral } \\
\text { vision (lift } \\
\text { eye) }\end{array}$ & marks & Y.Y &.$\leqslant 1$ & r... & 1.01 \\
\hline 4 & $\begin{array}{l}\text { Dynamic } \\
\text { visual } \\
\text { accuracy } \\
\text { (right hand) }\end{array}$ & marks & $1 . r$ & .19 & $1 \ldots$ & .07 \\
\hline 5 & $\begin{array}{l}\text { Dynamic } \\
\text { visual } \\
\text { accuracy (lift } \\
\text { hand) }\end{array}$ & marks & $.9 V$ &. .19 & $1 \ldots$ & .07 \\
\hline 6 & $\begin{array}{l}\text { side visual } \\
\text { Tracking }\end{array}$ & marks & $7.0 Y$ & .01 & V... & 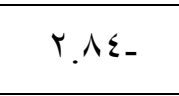 \\
\hline 7 & $\begin{array}{l}\text { Back visual } \\
\text { tracking }\end{array}$ & marks & 7. Y & .01 & $7 .$. & $1.0 \mathrm{~V}$ \\
\hline 8 & $\begin{array}{l}\text { sensory } \\
\text { perception } \\
\text { of the time } \\
(10 \mathrm{sec})\end{array}$ & second & 1.0 & $.1 \leqslant$ & $1 . V$ & $.01-$ \\
\hline 9 & $\begin{array}{l}\text { sensory } \\
\text { perception } \\
\text { of the time } \\
(25 \mathrm{sec})\end{array}$ & second & 1.17 & .10 & 1.19 & $.0 V_{-}$ \\
\hline 10 & $\begin{array}{l}\text { sensory } \\
\text { perception of } \\
\text { the time ( } 45 \\
\text { sec) }\end{array}$ & second & 1.10 & & 1.91 & $.0 V_{-}$ \\
\hline 11 & $\begin{array}{l}\text { sensory } \\
\text { perception of } \\
\text { distance }(5 \\
\mathrm{cm})\end{array}$ & $\mathrm{cm}$ & 1.1 & $\cdot Y$ & 1.14 & .00 \\
\hline
\end{tabular}

Assiut Journal For Sport Science Arts 
Follow Table (2)

Statistical characterization of the sample research in some visual abilities $\mathbf{n}=\mathbf{2 9}$

\begin{tabular}{|c|c|c|c|c|c|c|}
\hline Sr. & $\begin{array}{l}\text { The } \\
\text { variables }\end{array}$ & $\begin{array}{l}\text { Unit of } \\
\text { measuring }\end{array}$ & mean & Deviation & Median & skewness \\
\hline 12 & $\begin{array}{l}\text { sensory } \\
\text { perception } \\
\text { of distance } \\
(10 \mathrm{~cm})\end{array}$ & $\mathrm{cm}$ & $1 . r$. & $.1 \pi$ & 1.49 & $.1 \leq$ \\
\hline 13 & $\begin{array}{l}\text { sensory } \\
\text { perception } \\
\text { of distance } \\
(15 \mathrm{~cm})\end{array}$ & $\mathrm{cm}$ & $1.7 \mathrm{~V}$ & .11 & 1.71 & $\cdot 7 \leqslant$ \\
\hline 14 & $\begin{array}{l}\text { External } \\
\text { awareness }\end{array}$ & marks & $7 . \leqslant 1$ & .01 & ๆ. & $r . \varepsilon \Lambda$ \\
\hline 15 & $\begin{array}{l}\text { Level } \\
\text { performance }\end{array}$ & point & $0 . V Y$ &.$\vee \cdot$ & 7. . & $1.1 \Lambda_{-}$ \\
\hline
\end{tabular}

Table (2) that the skewness values are limited between (0.92: 1.59) and all values between \pm 3 , Which refers to the homogeneity of the research sample.

Table(3)

Valence between the control and experimental groups in the growth variables $n 1=n 2=12$

\begin{tabular}{|c|c|c|c|c|c|c|c|}
\hline \multirow[t]{2}{*}{ Sr. } & \multirow[t]{2}{*}{$\begin{array}{c}\text { The } \\
\text { variables }\end{array}$} & \multirow[t]{2}{*}{$\begin{array}{c}\text { Unit of } \\
\text { measuring }\end{array}$} & \multicolumn{2}{|c|}{ Control group } & \multicolumn{2}{|c|}{$\begin{array}{c}\begin{array}{c}\text { Experimental } \\
\text { group }\end{array} \\
\end{array}$} & \multirow[t]{2}{*}{$\begin{array}{c}\mathbf{T} \\
\text { value }\end{array}$} \\
\hline & & & mean & Deviation & mean & Deviation & \\
\hline 1 & $\overline{\text { age }}$ & year & 19.94 & .79 & $r \cdot .0$ & .01 &..$\leqslant 0$ \\
\hline$r$ & weight & $\mathrm{Kg}$ & Vo.TV & $\varepsilon . V \varepsilon$ & VT.YO & $\varepsilon . r$ & $\cdot \pi r$ \\
\hline$r$ & Tallness & $\mathrm{Cm}$ & $1 \vee 7 . .$. & r.vq & 180.11 & $\varepsilon . \mu 7$ & .00 \\
\hline
\end{tabular}

* The table value of $\mathrm{T}$. at the level of significance $0.05=2.07$

Table (3) shows that there are groups in the pre -measurement no differences with a statistical of growth variables, this refers significance between the to the valence of between the experimental and control two groups. 
Table (4)

Valence between the control and experimental groups in some visual abilities $\mathrm{n} 1=\mathrm{n} 2=12$

\begin{tabular}{|c|c|c|c|c|c|c|c|}
\hline \multirow{2}{*}{ Sr. } & \multirow{2}{*}{$\begin{array}{l}\text { The } \\
\text { variables }\end{array}$} & \multirow{2}{*}{$\begin{array}{l}\text { Unit of } \\
\text { measuring }\end{array}$} & \multicolumn{2}{|c|}{ Control group } & \multicolumn{2}{|c|}{$\begin{array}{l}\text { Experimental } \\
\text { group }\end{array}$} & \multirow{2}{*}{$\begin{array}{l}\mathbf{T} \\
\text { value }\end{array}$} \\
\hline & & & mean & Deviation & mean & Deviation & \\
\hline 1 & $\begin{array}{l}\text { visual reaction } \\
\text { time }\end{array}$ & marks & $10 . \leqslant r$ & .01 & س. &..$\leqslant 9$ & $\cdot \leqslant 1$ \\
\hline r & $\begin{array}{l}\text { Peripheral } \\
\text { vision (right } \\
\text { eye) }\end{array}$ & marks & Y.IV & $\cdot r q$ & r. ro &.$\leqslant 0$ & $\because \leqslant \wedge$ \\
\hline r & $\begin{array}{l}\text { Peripheral } \\
\text { vision } \quad \text { (lift } \\
\text { eye) }\end{array}$ & marks & Y.IV & $\cdot r q$ & r. ro &..$\leqslant 0$ & $\because \leqslant \wedge$ \\
\hline$\varepsilon$ & $\begin{array}{l}\text { Dynamic } \\
\text { visual } \\
\text { accuracy (right } \\
\text { hand) }\end{array}$ & marks & $1 \ldots$ & $\because \cdots$ & 1.1 & $\cdot r q$ & $1 . \cdots$ \\
\hline 0 & $\begin{array}{l}\text { Dynamic } \\
\text { visual } \\
\text { accuracy (lift } \\
\text { hand) }\end{array}$ & marks & $1 \ldots$ & $\because \cdots$ & $.9 r$ & $\cdot r q$ & $1 . \cdots$ \\
\hline 7 & $\begin{array}{l}\text { side visual } \\
\text { Tracking }\end{array}$ & marks & 7.0. &. or & 7.01 & .01 &. .49 \\
\hline v & $\begin{array}{ll}\text { Back visual } \\
\text { tracking }\end{array}$ & marks & Tr. & $\cdot . \leqslant 9$ & $T . \varepsilon Y$ & .01 & $\because \leqslant 1$ \\
\hline 8 & $\begin{array}{l}\text { sensory } \\
\text { perception of } \\
\text { the time (10 } \\
\text { sec) }\end{array}$ & second & $1 . \varepsilon$ & .10 & $1 . \varepsilon$ & .17 & .10 \\
\hline 9 & $\begin{array}{l}\text { sensory } \\
\text { perception of } \\
\text { the time ( } 25 \\
\text { sec) }\end{array}$ & second & 1.17 & .17 & 1.14 & $. .1 \leqslant$ & $\because \leqslant 7$ \\
\hline 10 & $\begin{array}{l}\text { sensory } \\
\text { perception of } \\
\text { the time (45 } \\
\text { sec) }\end{array}$ & second & 1.90 & $\cdot r^{\prime}$ & 1.91 & .19 & r \\
\hline 11 & $\begin{array}{l}\text { sensory } \\
\text { perception of } \\
\text { distance }(5 \mathrm{~cm})\end{array}$ & $\mathrm{cm}$ & 1.9 & $\because K r$ & 1.0 & 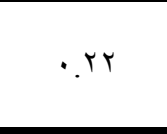 & $\because V$ \\
\hline
\end{tabular}

Assiut Journal For Sport Science Arts 
Follow Table (4)

Valence between the control and experimental groups in some visual abilities $\mathrm{n} 1=\mathrm{n} 2=12$

\begin{tabular}{|c|c|c|c|c|c|c|c|}
\hline \multirow{2}{*}{ Sr. } & \multirow{2}{*}{$\begin{array}{l}\text { The } \\
\text { variables }\end{array}$} & \multirow{2}{*}{$\begin{array}{l}\text { Unit of } \\
\text { measuring }\end{array}$} & \multicolumn{2}{|c|}{ Control group } & \multicolumn{2}{|c|}{$\begin{array}{l}\text { Experimental } \\
\text { group }\end{array}$} & \multirow{2}{*}{$\begin{array}{l}\mathbf{T} \\
\text { value }\end{array}$} \\
\hline & & & mean & Deviation & mean & Deviation & \\
\hline Ir & $\begin{array}{l}\text { sensory } \\
\text { perception of } \\
\text { distance } \\
\mathrm{cm}) \\
\end{array}$ & $\mathrm{cm}$ & $1 . r$. &. $.1 T$ & $1 . r \wedge$ & .17 & or \\
\hline r & $\begin{array}{l}\text { Sensory } \\
\text { perception of } \\
\text { distance } \quad(15 \\
\mathrm{cm})\end{array}$ & $\mathrm{cm}$ & $1 . V T$ & .10 & $1 . V r$ &. .17 &. .19 \\
\hline $1 \leq$ & $\begin{array}{l}\text { External } \\
\text { awareness }\end{array}$ & marks & 7.0. & $\because$ or & $T . \varepsilon r$ & .01 & $\cdot .99$ \\
\hline 10 & $\begin{array}{l}\text { Level } \\
\text { performance }\end{array}$ & point & $0 . \wedge r$ & .01 & $0.7 \mathrm{~V}$ & $\cdot \vee \wedge$ & $\cdot 7$ \\
\hline
\end{tabular}

The table value of $T$. at the level of significance $0.05=2.07$

Table (4) shows that there are no differences with a statistical significance between the experimental and control groups in the pre-measurement of some visual abilities, This refers to the valence of between the two groups .

Tests of visual abilities:

1. Test of Visual reaction time.

5. Sensory perception of the time.

2. Peripheral vision.

6. Sensory perception of distance.

3. Dynamic visual accuracy test. 7. External awareness

4. Side visual Tracking
Assessment the level performance of the basic skills: It was filmed the fireman carry skill of the sample research using a digital video camera, the first time in the pre- measurements and the second time in after measurements, was then display on the three Referees to assessment the technical performance level of the skills, so it gave 10 marks for each skill for assessment.

The proposed program of visual exercise:

The program contains exercises to improve visual abilities and specific exercise, and_drills to fireman carry in 
addition to specific warm-up is linked to the nature of the muscle work in the training modules and the end of its exercises to calm and organize to breathe and relax nervous and muscular to return to natural state . It was the drafting of training loads based on the limits of development the physical and skill level of specialized references, in addition to the results of measurements the survey study to determine the starting dose. The researchers have been applying the proposed training program for 12 weeks with three units per week on the experimental research sample; the control group has been applied to the same training program in relation to skill exercises for fireman carry with the exception of visual exercises part.

Study of basic research:

The researcher conducting the pre- measurement on day 4-5 / 2 / 2015 in Shiba youth center, and the proposed program (basic study) on experimental research sample in the period from $7 / 2 / 2015$ to 30 /4/ 2015at Shiba youth center, for (12) weeks rate of (3) units per week, The researcher conducting the after measurement on day 2-3 15/2015.

\section{Table (5)}

Significance of differences between pre and after measurement in some visual abilities of the control group $n=12$

\begin{tabular}{|c|c|c|c|c|c|c|c|}
\hline \multirow[t]{2}{*}{ Sr. } & \multirow{2}{*}{$\begin{array}{l}\text { The } \\
\text { variables }\end{array}$} & \multirow{2}{*}{$\begin{array}{l}\text { Unit of } \\
\text { measuring }\end{array}$} & \multicolumn{2}{|c|}{ measurement pre } & \multicolumn{2}{|c|}{$\begin{array}{l}\text { After } \\
\text { measurement }\end{array}$} & \multirow{2}{*}{$\begin{array}{l}\mathbf{T} \\
\text { value }\end{array}$} \\
\hline & & & Mean & Deviation & mean & Deviation & \\
\hline 1 & $\begin{array}{l}\text { visual } \\
\text { reaction } \\
\text { time }\end{array}$ & marks & $10 . \leqslant r$ & .01 & $10 . \wedge r$ & .01 & r.17 \\
\hline r & $\begin{array}{l}\text { Peripheral } \\
\text { vision (right } \\
\text { eye) }\end{array}$ & marks & T.IV &.$r q$ & $r . \varepsilon r$ & .01 & 1.94 \\
\hline$r$ & $\begin{array}{l}\text { Peripheral } \\
\text { vision (lift } \\
\text { eye) }\end{array}$ & marks & r.IV & $\cdot . r q$ & Tr.tr & $\cdot . \leqslant 9$ & $1 . \cdots$ \\
\hline$\varepsilon$ & $\begin{array}{l}\text { Dynamic } \\
\text { visual } \\
\text { accuracy } \\
\text { (right hand) }\end{array}$ & marks & $1 . \cdots$ & $\because \cdots$ & $1 . r 0$ & $\because \leqslant 0$ & 1.94 \\
\hline
\end{tabular}

Assiut Journal For Sport Science Arts 
Follow Table (5)

Significance of differences between pre and after measurement in some visual abilities of the control group $n=12$

\begin{tabular}{|c|c|c|c|c|c|c|c|}
\hline \multirow[t]{2}{*}{ Sr. } & \multirow{2}{*}{$\begin{array}{l}\text { The } \\
\text { variables }\end{array}$} & \multirow{2}{*}{$\begin{array}{l}\text { Unit of } \\
\text { measuring }\end{array}$} & \multicolumn{2}{|c|}{ measurement pre } & \multicolumn{2}{|c|}{$\begin{array}{l}\text { After } \\
\text { measurement }\end{array}$} & \multirow{2}{*}{$\begin{array}{l}\mathbf{T} \\
\text { value }\end{array}$} \\
\hline & & & Mean & Deviation & mean & Deviation & \\
\hline 0 & $\begin{array}{l}\text { Dynamic } \\
\text { visual } \\
\text { accuracy } \\
\text { lift hand) }\end{array}$ & marks & $1 . \cdots$ & $\because \cdots$ & $1.1 \mathrm{~V}$ & $\cdot . r 9$ & $1 . \leqslant 1$ \\
\hline 7 & $\begin{array}{l}\text { side visual } \\
\text { Tracking }\end{array}$ & marks & 7.0. & $.0 r$ & ד. & $\cdot V T$ & *r.ro \\
\hline$\checkmark$ & $\begin{array}{l}\text { Back visual } \\
\text { tracking }\end{array}$ & marks & T.r & $\because \leqslant 9$ & 7.0. & $\cdot .7 V$ & $1 . \leqslant 1$ \\
\hline 8 & $\begin{array}{l}\text { sensory } \\
\text { perception } \\
\text { of the time( } \\
10 \mathrm{sec})\end{array}$ & second & $1 . \cdot \varepsilon$ & .10 & $1 . \cdot r$ & .17 & 1.71 \\
\hline 9 & $\begin{array}{l}\text { sensory } \\
\text { perception } \\
\text { of the time( } \\
25 \mathrm{sec})\end{array}$ & second & 1.17 & .17 & $1.1 \leq$ & $\because \mathrm{VV}$ & $\cdot 9 V$ \\
\hline 10 & $\begin{array}{l}\text { sensory } \\
\text { perception } \\
\text { of the time( } \\
45 \mathrm{sec})\end{array}$ & second & 1.90 & $\cdot Y_{I}$ & 1.94 & זr & $1 . Y_{1}$ \\
\hline 11 & $\begin{array}{l}\text { sensory } \\
\text { perception of } \\
\text { distance }(5 \\
\mathrm{cm})\end{array}$ & $\mathrm{cm}$ & 1.9 & $\cdot Y r$ & 1.0 & .17 & . r \\
\hline 12 & $\begin{array}{l}\text { sensory } \\
\text { perception } \\
\text { of distance } \\
(10 \mathrm{~cm})\end{array}$ & $\mathrm{cm}$ & $1 . r$. &. $.1 T$ & $1 . V T$ & .10 & HוT \\
\hline 13 & $\begin{array}{l}\text { sensory } \\
\text { perception } \\
\text { of distance } \\
(15 \mathrm{~cm})\end{array}$ & $\mathrm{cm}$ & $1 . V T$ & .10 & 1.79 & .10 & r.ir \\
\hline 14 & $\begin{array}{l}\text { External } \\
\text { awareness }\end{array}$ & marks & 7.0. & $.0 r$ & 7.10 & $\cdot$. vo & 1.94 \\
\hline 15 & $\begin{array}{l}\text { Level } \\
\text { performance }\end{array}$ & point & $0 . \wedge \mu$ & .01 & 7.1 & $\cdot .49$ & 1.49 \\
\hline
\end{tabular}

The table value of $\mathrm{T}$. at the level of significance $0.05=2.20$ 
Table (5) There are no significant differences between pre and after measurement in some visual abilities and level performance expect side visual

Table (6)

Significance of differences between pre and after measurement in some visual abilities of the experimental group $n=12$

\begin{tabular}{|c|c|c|c|c|c|c|c|}
\hline \multirow[t]{2}{*}{ Sr. } & \multirow{2}{*}{$\begin{array}{l}\text { The } \\
\text { variables }\end{array}$} & \multirow{2}{*}{$\begin{array}{l}\text { Unit of } \\
\text { measuring }\end{array}$} & \multicolumn{2}{|c|}{ measurement pre } & \multicolumn{2}{|c|}{$\begin{array}{l}\text { After } \\
\text { measurement }\end{array}$} & \multirow{2}{*}{$\begin{array}{l}\text { T } \\
\text { value }\end{array}$} \\
\hline & & & Mean & Deviation & mean & Deviation & \\
\hline 1 & $\begin{array}{l}\text { visual } \\
\text { reaction } \\
\text { time }\end{array}$ & marks & אח. 10 & $\because \leqslant 9$ & $19 . \wedge r$ & $1.7 \varepsilon$ & $* 9.7$. \\
\hline r & $\begin{array}{l}\text { Peripheral } \\
\text { vision (right } \\
\text { eye) }\end{array}$ & marks & r. ro & $\because \leqslant 0$ & $\varepsilon .1 V$ & 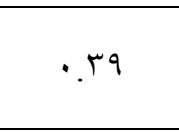 & $* 1$ r.^9 \\
\hline r & $\begin{array}{l}\text { Peripheral } \\
\text { vision (lift } \\
\text { eye) }\end{array}$ & marks & r. ro & $\because \leqslant 0$ & r.Ar & $\because V Y$ & $* \wedge . Y$. \\
\hline$\varepsilon$ & $\begin{array}{l}\text { Dynamic } \\
\text { visual } \\
\text { accuracy } \\
\text { (right hand) }\end{array}$ & marks & 1.1 &.$r q$ & מז. & $\because \leqslant 9$ & $* 9.0 \mathrm{~V}$ \\
\hline 0 & $\begin{array}{l}\text { Dynamic } \\
\text { visual } \\
\text { accuracy } \\
\text { (lift hand) }\end{array}$ & marks & $.9 r$ &.$r q$ & I.Ar &. .99 & $* 7.1 \mathrm{~V}$ \\
\hline 7 & $\begin{array}{l}\text { side visual } \\
\text { Tracking }\end{array}$ & marks & 7.01 & .01 & $\wedge .01$ & .01 & $* 11 . \leqslant 9$ \\
\hline V & $\begin{array}{l}\text { Back visual } \\
\text { tracking }\end{array}$ & marks & $T . \varepsilon r$ & .01 & $\vee .0 \Lambda$ & .01 & $* 1 . r$ N \\
\hline 8 & $\begin{array}{l}\text { sensory } \\
\text { perception } \\
\text { of the time } \\
(10 \mathrm{sec})\end{array}$ & second & $1 . \varepsilon$ & .17 &.$v 1$ & r & $* 17.7 \varepsilon$ \\
\hline 9 & $\begin{array}{l}\text { sensory } \\
\text { perception } \\
\text { of the time( } \\
25 \mathrm{sec})\end{array}$ & second & 1.11 & $.1 \leq$ & $.9 r$ & $.1 r$ & $* 11$. . \\
\hline
\end{tabular}

Tracking and sensory perception of the time (10 sec) of the control group for after measurement. 
Follow Table (6)

Significance of differences between pre and after measurement in some visual abilities of the experimental group $n=12$

\begin{tabular}{|c|c|c|c|c|c|c|c|}
\hline \multirow{2}{*}{ Sr. } & \multirow{2}{*}{$\begin{array}{l}\text { The } \\
\text { variables }\end{array}$} & \multirow{2}{*}{$\begin{array}{l}\text { Unit of } \\
\text { measuring }\end{array}$} & \multicolumn{2}{|c|}{ measurement pre } & \multicolumn{2}{|c|}{$\begin{array}{l}\text { After } \\
\text { measurement }\end{array}$} & \multirow{2}{*}{$\begin{array}{l}\mathbf{T} \\
\text { value }\end{array}$} \\
\hline & & & Mean & Deviation & mean & Deviation & \\
\hline 10 & $\begin{array}{l}\text { sensory } \\
\text { perception } \\
\text { of the time( } \\
45 \mathrm{sec})\end{array}$ & second & 1.91 & .19 & $1.7 \pi$ & .19 & $* 1 . \leqslant 9$ \\
\hline 11 & $\begin{array}{l}\text { sensory } \\
\text { perception } \\
\text { of } \\
\text { distance }(5 \\
\mathrm{cm})\end{array}$ & $\mathrm{cm}$ & 1.0 & $\cdot r r$ & $\cdot \wedge$. & .19 & $* 10.74$ \\
\hline 12 & $\begin{array}{l}\text { sensory } \\
\text { perception } \\
\text { of distance } \\
(10 \mathrm{~cm})\end{array}$ & $\mathrm{cm}$ & $1 . \mathrm{r}$ & .17 & 1.71 & $\because$ ro & $* 7 . .0$ \\
\hline 13 & $\begin{array}{l}\text { sensory } \\
\text { perception } \\
\text { of distance } \\
(15 \mathrm{~cm})\end{array}$ & $\mathrm{cm}$ & $1 . v r$ & .17 & $1 . \varepsilon$ & .11 & $* \vee .99$ \\
\hline 14 & $\begin{array}{l}\text { External } \\
\text { awareness }\end{array}$ & marks & $T . \varepsilon Y$ & $\because 01$ & 1.TV & $\because \leqslant 9$ & $* I V . Y T$ \\
\hline 15 & $\begin{array}{l}\text { Level } \\
\text { performance }\end{array}$ & point & $0.7 \mathrm{~V}$ & $\cdot \vee \wedge$ & 1.0. & $1.1 \mathrm{~V}$ & *YO. Y r \\
\hline
\end{tabular}

The table value of $\mathrm{T}$. at the level of significance $0.05=2.20$

Table (6) there are all variables of the significant differences between experimental group for after pre and after measurement in measurement.

Table (7)

Significance of differences between control and experimental groups in visual abilities $n 1=n 2=12$

\begin{tabular}{|c|c|c|c|c|c|c|c|}
\hline \multirow[t]{2}{*}{ Sr. } & \multirow{2}{*}{$\begin{array}{l}\text { The } \\
\text { variables }\end{array}$} & \multirow{2}{*}{$\begin{array}{l}\text { Unit of } \\
\text { measuring }\end{array}$} & \multicolumn{2}{|c|}{ Control group } & \multicolumn{2}{|c|}{$\begin{array}{l}\text { experimental } \\
\text { group }\end{array}$} & \multirow{2}{*}{$\begin{array}{l}\text { T } \\
\text { value }\end{array}$} \\
\hline & & & mean & Deviation & mean & Deviation & \\
\hline 1 & $\begin{array}{l}\text { visual } \\
\text { reaction time }\end{array}$ & marks & $10.1 \mu$ & .01 & $19 . \wedge r$ & $1.7 \varepsilon$ & $* \vee .97$ \\
\hline r & $\begin{array}{l}\text { Peripheral } \\
\text { vision (right } \\
\text { eye ) }\end{array}$ & marks & $r . \varepsilon r$ & .01 & $\varepsilon .1 V$ & $\cdot r q$ & $* 9.49$ \\
\hline
\end{tabular}

Follow Table (7)

Assiut Journal For Sport Science Arts 
Significance of differences between control and experimental groups in visual abilities $n 1=n 2=12$

\begin{tabular}{|c|c|c|c|c|c|c|c|}
\hline \multirow{2}{*}{ Sr. } & \multirow{2}{*}{$\begin{array}{l}\text { The } \\
\text { variables }\end{array}$} & \multirow{2}{*}{$\begin{array}{l}\text { Unit of } \\
\text { measuring }\end{array}$} & \multicolumn{2}{|c|}{ Control group } & \multicolumn{2}{|c|}{$\begin{array}{l}\text { experimental } \\
\text { group }\end{array}$} & \multirow{2}{*}{$\begin{array}{l}\text { T } \\
\text { value }\end{array}$} \\
\hline & & & mean & Deviation & mean & Deviation & \\
\hline r & $\begin{array}{l}\text { Peripheral } \\
\text { vision(lift } \\
\text { eye) }\end{array}$ & marks & rr. & $\because \leqslant 9$ & r.Ar & $\because V Y$ & $* 0.9 V$ \\
\hline$\varepsilon$ & $\begin{array}{l}\text { Dynamic } \\
\text { visual } \\
\text { accuracy } \\
\text { (right hand) }\end{array}$ & marks & 1.10 &.$\leqslant 0$ & זr. & $\because \leqslant 9$ & $* 0.71$ \\
\hline 0 & $\begin{array}{l}\text { Dynamic } \\
\text { visual } \\
\text { accuracy (lift } \\
\text { hand) }\end{array}$ & marks & 1.18 &.$r q$ & I.A &.$r q$ & $* \varepsilon . Y$. \\
\hline 7 & $\begin{array}{l}\text { side visual } \\
\text { Tracking }\end{array}$ & marks & &.$V Y$ & 1.01 & .01 & $*\rceil . \wedge 7$ \\
\hline V & $\begin{array}{l}\text { Back visual } \\
\text { tracking }\end{array}$ & marks & 7.0. & $.7 V$ & $\vee .01$ & .01 & $* \varepsilon . \leqslant r$ \\
\hline 8 & $\begin{array}{l}\text { sensory } \\
\text { perception of } \\
\text { the time( } 10 \\
\text { sec) }\end{array}$ & second & $1 . r$ & .17 &.$v 1$ & $.1 T$ & *0. T \\
\hline 9 & $\begin{array}{l}\text { sensory } \\
\text { perception of } \\
\text { the time( } 25 \\
\text { sec) }\end{array}$ & second & $1.1 \leq$ & $.1 \mathrm{~V}$ & $.9 r$ & .14 & *Y. \\
\hline 10 & $\begin{array}{l}\text { sensory } \\
\text { perception of } \\
\text { the time( } 45 \\
\text { sec) }\end{array}$ & second & 1.94 & זr. & 1.7 & .19 & *r.vo \\
\hline 11 & $\begin{array}{l}\text { sensory } \\
\text { perception of } \\
\text { distance }(5 \mathrm{~cm})\end{array}$ & $\mathrm{cm}$ & 1.0 & .17 & $\because \wedge$. & .19 & *r.01 \\
\hline 12 & $\begin{array}{l}\text { sensory } \\
\text { perception of } \\
\text { distance }(10 \mathrm{~cm})\end{array}$ & $\mathrm{cm}$ & I.VY & .10 & 1.71 &. ro & $* . . \leqslant 1$ \\
\hline 13 & $\begin{array}{l}\text { sensory } \\
\text { perception of } \\
\text { distance }(15 \mathrm{~cm})\end{array}$ & $\mathrm{cm}$ & 1.79 & .10 & $1 . \varepsilon$. & .11 & *0.r \\
\hline 14 & $\begin{array}{l}\text { External } \\
\text { awareness }\end{array}$ & marks & 7.10 &. vo & A. $7 \mathrm{~V}$ &.$\leqslant 9$ & $* V . \mu V$ \\
\hline 15 & $\begin{array}{l}\text { Level } \\
\text { performance }\end{array}$ & point & 7.11 &.$r q$ & 1.0. & $1.1 \mathrm{~V}$ & $* 7.97$ \\
\hline
\end{tabular}

The table value of $T$. at the level of significance $0.05=2.07$ 
Table (7) there are statistically significant
difference between after measurement for the control

group and the experimental groups in favor of the experimental group in all variables.

\section{Table (8)}

Percentage improvement in the in some visual abilities of the two groups (control group and the experimental group) $n 1=n 2=12$

\begin{tabular}{|c|c|c|c|c|c|c|c|}
\hline \multirow[b]{2}{*}{ Sr. } & \multirow{2}{*}{$\begin{array}{l}\text { The } \\
\text { variables }\end{array}$} & \multicolumn{2}{|c|}{$\begin{array}{l}\text { control } \\
\text { group }\end{array}$} & \multirow{2}{*}{$\begin{array}{l}\text { Percentage } \\
\text { improvement } \\
\%\end{array}$} & \multicolumn{2}{|c|}{$\begin{array}{l}\text { experimental } \\
\text { group }\end{array}$} & \multirow{2}{*}{$\begin{array}{l}\text { Percentage } \\
\text { improvement } \\
\%\end{array}$} \\
\hline & & $\begin{array}{l}\text { Mean } \\
\text { of } \\
\text { pre }\end{array}$ & $\begin{array}{l}\text { Mean } \\
\text { of } \\
\text { after }\end{array}$ & & $\begin{array}{l}\text { Mean } \\
\text { of } \\
\text { pre }\end{array}$ & $\begin{array}{l}\text { Mean } \\
\text { of } \\
\text { after }\end{array}$ & \\
\hline 1 & $\begin{array}{l}\text { visual } \\
\text { reaction } \\
\text { time } \\
\end{array}$ & $10 . \leqslant Y$ & 10.14 & r.v. & س & $19 . \wedge r$ & rq.ro \\
\hline 2 & $\begin{array}{l}\text { Peripheral } \\
\text { vision } \\
\text { right eye ) }\end{array}$ & Y.IV & $r . \leqslant Y$ & $11.0 \leqslant$ & T. YO & $\varepsilon .1 V$ & 10.19 \\
\hline 3 & $\begin{array}{l}\text { Peripheral } \\
\text { vision( lift } \\
\text { eye ) }\end{array}$ & Y.IV & Tr.r & $v .79$ & r. ro & r.یr & $v \cdot r v$ \\
\hline 4 & $\begin{array}{l}\text { Dynamic } \\
\text { visual } \\
\text { accuracy ( } \\
\text { right hand) }\end{array}$ & $1 . \cdots$ & $1 . r_{0}$ & ro... & $1 . \cdot 1$ & Tr.rT & 110.49 \\
\hline 5 & $\begin{array}{l}\text { Dynamic } \\
\text { visual } \\
\text { accuracy } \\
\text { lift hand) }\end{array}$ & $1 . \cdots$ & $1.1 \mathrm{~V}$ & $17.7 \mathrm{~V}$ & .94 & $1 . \wedge r$ & $1 \ldots$. \\
\hline 6 & $\begin{array}{l}\text { side visual } \\
\text { Tracking }\end{array}$ & 7.0. & \urcorner.$\wedge r$ & 0.11 & 7.01 & 1.01 & $r$ r. \\
\hline 7 & $\begin{array}{l}\text { Back visual } \\
\text { tracking }\end{array}$ & Tr. & 7.0. & r.t & $7 . \leqslant Y$ & $V .01$ & 11.11 \\
\hline 8 & $\begin{array}{l}\text { sensory } \\
\text { perception } \\
\text { of the time( } \\
10 \mathrm{sec})\end{array}$ & $1 . \varepsilon$ & 1.4 & T.AV & $1 . \cdot \varepsilon$ & 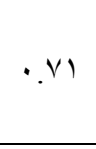 & TI. TV \\
\hline 9 & $\begin{array}{l}\text { sensory } \\
\text { perception } \\
\text { of the time( } \\
25 \mathrm{sec})\end{array}$ & 1.17 & $1.1 \leq$ & I.rV & 1.14 & .94 & 11.11 \\
\hline
\end{tabular}


Follow Table (8)

Percentage improvement in the in some visual abilities of the two groups (control group and the experimental group) $n 1=n 2=12$

\begin{tabular}{|c|c|c|c|c|c|c|c|}
\hline \multirow[b]{2}{*}{ Sr. } & \multirow[b]{2}{*}{$\begin{array}{l}\text { The } \\
\text { variables }\end{array}$} & \multicolumn{2}{|c|}{$\begin{array}{l}\text { control } \\
\text { group }\end{array}$} & \multirow{2}{*}{$\begin{array}{l}\text { Percentage } \\
\text { improvement } \\
\%\end{array}$} & \multicolumn{2}{|c|}{$\begin{array}{l}\text { experimental } \\
\text { group }\end{array}$} & \multirow{2}{*}{$\begin{array}{l}\text { Percentage } \\
\text { improvement } \\
\%\end{array}$} \\
\hline & & $\begin{array}{l}\text { Mean } \\
\text { of } \\
\text { pre }\end{array}$ & $\begin{array}{l}\text { Mean } \\
\text { of } \\
\text { after }\end{array}$ & & $\begin{array}{l}\text { Mean } \\
\text { of } \\
\text { pre }\end{array}$ & $\begin{array}{l}\text { Mean } \\
\text { of } \\
\text { after }\end{array}$ & \\
\hline 10 & $\begin{array}{l}\text { sensory } \\
\text { perception } \\
\text { of the time( } \\
45 \mathrm{sec})\end{array}$ & 1.90 & $1.9 r$ & $1 . \leqslant 1$ & 1.91 & 1.7 & $1 V . \leqslant 0$ \\
\hline 11 & $\begin{array}{l}\text { sensory } \\
\text { perception } \\
\text { of } \\
\text { distance }(5 \\
\mathrm{cm})\end{array}$ & 1.7 & 1.0 & $1 . r$ & 1.0 &.$\wedge$. & $r \leq . r)$ \\
\hline 12 & $\begin{array}{l}\text { sensory } \\
\text { perception } \\
\text { of distance } \\
(10 \mathrm{~cm})\end{array}$ & $1 . r$. & $1 . V Y$ & rI.9r & $1 . r \Lambda$ & 1.71 & r. \\
\hline 13 & $\begin{array}{l}\text { sensory } \\
\text { perception } \\
\text { of distance } \\
(15 \mathrm{~cm})\end{array}$ & $1 . V Y$ & 1.79 & $1 . v$. & l.VT & $1 . \varepsilon$ & 11.94 \\
\hline 14 & $\begin{array}{l}\text { External } \\
\text { awareness }\end{array}$ & 7.0. & 7.10 & r.Ao & $7 . \leqslant Y$ & ᄉ.TV & ro. T \\
\hline 15 & $\begin{array}{l}\text { Level } \\
\text { performance }\end{array}$ & $0 . \wedge T$ & $7 . \wedge$ & $\varepsilon . r q$ & $0.7 \mathrm{~V}$ & 1.0. & $0 . . \cdots$ \\
\hline \multicolumn{8}{|c|}{ 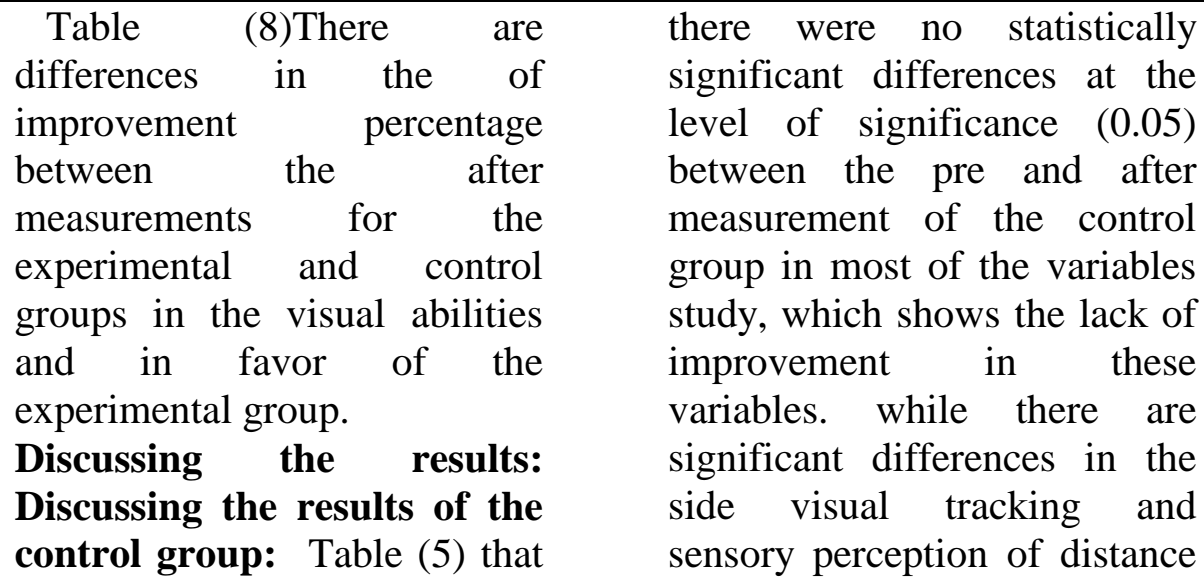 } \\
\hline
\end{tabular}

Assiut Journal For Sport Science Arts 
$(10 \mathrm{~cm})$, which indicates the improvement of those variables . the researchers attributed to that the impact of continuous training on the visual abilities for the program. where the control group came under the same of drills exercise to fireman carry without visual exercises which led to a relative improvement in the side visual tracking and sensory perception of distance $(10 \mathrm{~cm}), \quad$ however, this improvement was automatically and It does not compare the improvement that has been intentionally through the training program applied to the experimental group, the researchers attributed to that the lack of interest wrestlers visual abilities and the quality and efficiency of performance and attention to increasing the number of attempts performance only.

Discussing the results of the experimental group: Table (6) that there are significant differences between the pre and after measurement and in favor of experimental group in all the variables, which shows the experimental group improved under the proposed training program, which includes all the visual exercises, that important variable in the implementation and performance of fireman carry skill and the level performance for wrestlers, Because if the attacker has improved on the visual reaction time, peripheral vision, the visual tracking, sensory perception of the time and distance and increase the external awareness of the external environment and defender, Lack of time it takes for wrestlers to hear the referee's whistle to hold defender and lift it and the more successful in the performance and implementation of the fireman carry skill, an increase in the economy of effort and time wrestler, these results are consistent with the study of "Doaa Mahmoud" (2002) (3), Milslajl Millslagle (2004) (19), "Ashraf khtaab, Mervat Rashad" (2005) (13 ), " Thomas and others "(2005) (21), Magda Ismail and Others" (2007) (8), "Hoda Saber" (2008) (6), "Najla Tunahi" (2009) (11), , the results of these studies to the active role of visual abilities and improve the physical level to wrestlers, and therefore the positive impact on the 
development of level performance skills and this is consistent with the study of "Asseman F. (14), Hendri B." (16) in that they have Proved that visual abilities are similar to the physical abilities that can be training and development, and It is not linked to strongly visual only and that is essential, but the extent of the possibility of athlete to use information obtained through the eye inside the mat optimal use, " Barry seiller" (2004 ) (15) indicates that the visual variables have a positive and direct impact on the level of performance, athletics have a distinctive visual abilities nonathletes.

And consistent both, "Hitzeman \& Beckerman" (1993) (17), the visual abilities can be trained and developed and improved by the visual training, which are important for athletes, it has been proven that the visual training moves its effect on athletic performance and also to nonathletes as theses visual abilities is directly linked to improved athletic performance.

Accordingly, the results of the present study indicate that there are statistically significant differences between the averages of pre and after measurements in some visual abilities and level performance of fireman carry skill for the experimental group in favor of the after measurement, which achieves the first hypothesis search.

The results of the table ( $\mathrm{V}$ ) to improve after measurement for experimental sample about control sample in all search variables, and the researchers attributed to this improvement to undergo the experimental group of visual exercises within the proposed training program, because if the attacker has improved on the visual reaction time, peripheral vision, the visual tracking, sensory perception of the time and distance and increase the external awareness of the external environment and defender, lack of time it takes for wrestlers to hear the referee's whistle to hold defender and lift it and the more successful in the performance and implementation of the fireman carry skill, an increase in the economy of effort and time wrestler, these results are consistent with the study of Ahmed farouk (2008)(2) , Gihan Mohamed(2005) (5), 
Abdel halem fathi (1994) (1), Laila refaat (2009) (7), Mohamed saeed (2011) (10), Mahmoud abdel mohesen (2010)(9) , These results indicated that improving the level of some visual capabilities improve the level of performance skills to the experimental group , and improved wrestler in response to the referee's whistle, and improved speed of catch contender, and then lifted him and completing fireman carry skill, increasing the number of technical points which gets them depends on both the number of movements that performed and technical points due for every movement, this means that the increased number of technical points happened to increase the efficiency of the wrestler from the above researchers have concluded that there are statistically significant differences between the averages of after measurements for the experimental and control groups in some visual Abilities and level performance of fireman carry skill and in favor of the experimental group, which achieves the second hypothesis search.
Table (^) improved the experimental group on the control rates in all the variables under study, the researchers attributed the improvement in the experimental group from the control group in all search variables under study to proposed training program, which aims to use visual exercises as one of the most influential variables on the level performance of wrestler and get the technical points to win the round and the match, and the use of the program for different types of resistors and weightlifting, and taking into account individual differences among wrestlers in different physical style , and appropriateness of the training load for the experimental group , which had an impact in improving the physical level and the skill and there are differences in improvement ratios between experimental and control groups in some visual abilities and level performance of fireman carry skill and in favor of the experimental group, which achieves the third hypothesis search.

Barry seiller $(Y \ldots \varepsilon)(10)$ refer to The visual exercises are considered an integral part 
of the overall sports training programs, and good vision and eye muscle movement with the ability to focus all of which helps to improve athletic performance level .

\section{Conclusions: The}

1. The use of visual exercises used in the research to a marked improvement in visual capacity and the level of performance of fireman carry with the experimental group. 2. There are differences in the rates of improvement between the experimental and control groups in favor of the experimental group in all variables search.

\section{Recommendations:}

1. Attention to the development of the visual abilities of wrestlers.

2. Training on development the visual capacity an essential part of the sporting preparation for junior and adult programs. 3 . The importance of the visual exercises related to the nature of sports.

4. Attention to conduct special visual abilities measurements on a regular basis with the physical and technical skills and tactical measurements before, during and after the training season for the players.

5. Further research is related to the visual training to other samples of the wrestlers. 6. Guide trainers proposed exercises in this research and try to apply them.

The references: The Arabic references

\section{Abdel Halim Fathi Abdel}

Halim (1994): visual perception and spatial cognition relationship Al_husakinesthetic-fixing in wrestling, Master Thesis, Faculty of Physical Education, Minia University.

\section{Ahmed Farouk Khalaf} (2008): The impact of the optical vision program on the level performance of some visual and skills variables of basketball players, the fourth regional conference of the International Council for Health, Physical Education, Recreation and Sports and expression motor for the Middle East, Part 4, Faculty of Physical Education - Abu Qir, Alexandria University, Egypt 15-17 October.

\section{Doaa Mohamed}

Mahmoud2002: the impact of a program to train the eye in the fencing and its impact on some physiological variables associated with tension and the level of performance, Ph.D. thesis, Faculty of Physical 
Education for Girls, Helwan University.

\section{El said Ali Ahmed, Faika} Mohammed Badr (2001): visual Perceptual and audiovisual,el nahda el-masria Library, Cairo.

\section{5-Gihan Mohammed Fouad,} Iman Abdullah Zaid (2005): the effectiveness of visual training on some skill variables and visual capabilities in volleyball, global education magazine, Volume 2, College of Education, Sports Girls, Zagazig University.

6. Hoda Hassan Saber (2008): The effect of the impact of use visual skills to improve scoring accuracy and focus attention to the players kumite, Master, Faculty of Physical Education for Girls, Zagazig University.

7. Laila rafeat Ahmed (2009): The effect of visual exercises on some skills and visual cognitive abilities and level performance skills of the players volleyball, Journal of Sports Science and Art, Volume 32, Series 2, the Faculty of Physical Education for Girls, helwan University.

8. Magda Mustafa Ismail et al. (2006): effective visual training program on some visual skills, visual cognitive skills and relationship to the level of performance of some of the control skills of the junior rhythmic gymnastics, Journal Comprehensive Education, College of Physical Education, Girls, Zagazig University.

\section{Mahmoud abd el Mohsen}

Naji (2008): The effect of vision training on the performance of the free defender in volleyball, Ph.D. thesis, Faculty of Physical Education,

Minia University.

\section{Mohamed Saeed Abu} Nour (2009): The effect of the development of some of the visual capabilities on effectiveness of the counterattack for kumite players in karate sport, Faculty of Physical Education for Boys, Zagazig University, 2011.

\section{Najlaa amin Tunahi} (2010): The effect of development the specfic visual skills on certain some physical variables and chemicals vector and their relationship effectively tactical activity during kumite games, magazine theories and applications, Series68, Faculty of Physical Education for Boys in $\mathrm{Abu}$ Qir, Alexandria University. 


\section{Zaki Mohammed Hassan} (2002): Methods of teaching volleyball (teaching- trainingevaluation) el-ashaa Library, Alexandria.

\section{Foreign References:}

1r. Ashraf K., Merfat R. (2005): Aspisific visual skills training programme improves fencing performance level, visual variables and static balance, 10th Annval congress of European college of sport science Belgrade, 13-16 July.

14. Asseman F, Caron O, Cremieux J, (2002): Effect of the removal of vision on body sway during different postures in elite gymnastics, Int. Sports med., Mar, 26, 116-11.

15. Barry L. seiller (2004) : visual skills and volley ball, visual performance center ceorgia Tech Athletic Association visual fitness Institute , Oct 29.

16. Hendri Buys (2002): The development of norms and protocol in sports vision evaluation, magister philosphiae, faculty of science Rand Afrikaans, university. 17. Hitzeman SA \& Beckrman SA (1993): What the literature says about sports vision, a service of national library of medicine and the national institutes of health pmid: 83243222 (pubmed indexed for Medline).

18. Isabel walker (2001): Why visual training programmes for sport don't work, sports sic, Mar 19 (3) p203-220.

19. Millsagle, D (2004): Coincidence anticipation and Dynamic visual acurancy in young adolescents., percept mot skills. Dec; 99 (3pt2):1147-1156.

20. Suzanna cathrina vente (2003): A comparison of the visual skills of two different age group high school rugby players, master philosophiae, faculty of science, Rand Afikaans university.

21. Tomas L. et al (2005): Visual evoked potentials reaction tines and of the wit waters and Johannesburg, South Africa, Journal of eye dominance in cricketers university, sports medicine and physical fitness. Vol. 4. 